\title{
A Source-Destination Network Coded Cooperation for Wireless Ad-hoc Networks
}

\author{
Kaibin Zhang ${ }^{1}$, Liuguo Yin ${ }^{2}$, Jianhua $\mathrm{Lu}^{1,2}$ \\ ${ }^{1}$ Department of Electronic Engineering ${ }^{2}$ School of Aerospace \\ Tsinghua University \\ Beijing 100084, P.R.China \\ E-mail: zhangkb@wmc.ee.tsinghua.edu.cn
}

\begin{abstract}
Source-destination network coded cooperation (SDNCC), an extension of adaptive network coded cooperation (ANCC), which uses network coding and matches code-ongraph with network-on-graph, is proposed for wireless ad-hoc networks that comprise a collection of terminals communicating wirelessly to a common destination. In SDNCC, the destination broadcasts which terminal packets to be selected in the relay phase, which generates low-density generator-matrix (LDGM) codes with unequal error protection at cost of 1 bit per terminal. Additionally, the outage probability of SDNCC is evaluated and closed-form expression is derived for infinity networks. Furthermore, simulation results show that SDNCC achieves $4 \mathrm{~dB}$ performance improvement over ANCC at frame error ratio (FER) of $3 \times 10^{-5}$.
\end{abstract}

Index Terms-wireless ad-hoc network, network coding, lowdensity generator-matrix (LDGM), distributed coding

\section{INTRODUCTION}

Transmit diversity, a useful technique for combating random fading, may not be applicable to ad-hoc wireless networks due to size, complexity, or hardware limitations. The idea of user cooperation [1] involves the deliberate permission of one or more cooperating nodes, known as the relays, into the conventional point-to-point communication link. Thereby, user cooperation can create transmit diversity and improve capacity and outage probability in wireless systems.

Combining network coding [2][3] with user cooperation, adaptive network coded cooperation (ANCC) [4][5] has been one of the most valuable works recently. Matching the instantaneous network topologies, ANCC adaptively generates an ensemble of distributed low-density parity-check (LDPC) codes [7] at the destination. Using belief propagation (BP) decoding algorithm at the destination, ANCC obtains much more performance improvement over the repetition schemes [8] and Space-Time Coded Cooperation (STCC) frameworks [9]. However, ANCC protocol permits all terminal packets to be selected for computing check-sums and all the terminals to relay check-sums, which protects all the terminals equally. Clearly and intuitively, system should provide more protection to terminals with poor source-destination channel to improve the performance. Hence, duo to only considering the terminalterminal cooperation rather than overall cooperation, ANCC which doesn't use the dimension of the source-destination channel, is a suboptimal solution to the end-to-end performance.
Integrated cooperation means pulling together all dimensions of resources [3][10] among different users, such as time, frequency, spatial or terminals, etc. Recently many papers which allow users to exchange simple information have appeared [11]-[13]. References [11][12] analyze the performance of opportunistic relaying protocols that employ simple feedback from the receivers, but network coding is not adopted. Another study proposes an opportunistic networkcoded cooperation scheme for small networks [13]. In this paper, we propose source-destination network coded cooperation (SDNCC) to achieve a potential larger diversity gain of large wireless networks. Compared with ANCC, SDNCC adds indication phase that the destination broadcasts the message that only the packets in first $l$ worst source-destination channels need to be selected for check-sum, which costs 1 bit per terminal. Thus, matching channel quality, LDPC codes with unequal error protection are generated in an efficient, practical and distributed manner.

Intuitively, indication phase helps SDNCC make efficient use of the degree of freedom of the channel specially at high SNR. Obviously, ANCC is a special case of SDNCC when $l$ is equal to the terminal number $m$. Moreover, when $m$ approaches infinity, closed-form expressions for outage probability is derived for SDNCC. For any finite network, we perform its numerical evaluation. From the analysis results, the outage probability of SDNCC improves with the decrease of $l$. Whether analysis or simulation results, SDNCC has superior performance over ANCC.

The rest of the paper is organized as follows: Section II describes the system model of interest. Section III briefs ANCC. Section IVprovides SDNCC protocol, and Section V analyzes the outage probability and provides the simulation for SDNCC . Finally, Section VI draws the conclusion.

\section{SYSTEM MODEL}

The system model of interest comprises $m$ terminals wirelessly sending data to a common destination. We consider all channels suffer from frequency nonselective fading and are spatially independent and orthogonal in frequency, time or spread code. The channel noise $Z$ accounts for the addictive channel noise and inference, which is modeled as a complex Gaussian random variable with variance $N_{0}$. The fading coefficient $h$ is modeled as a zero-mean, independent, circularly 
complex Gaussian random variable with unit variance. The magnitude $|h|$ is Rayleigh distributed and the probability density function (pdf) of the channel power $\mu=|h|^{2}$ is $p_{u}(x)=e^{-x}$.

For simplicity, we assume all the communication channels, either between terminals or from a terminal to destination, have the same signal-to-noise ratio (SNR). We also assume that only receivers know the channel state information (CSI). Furthermore, we consider that the fading coefficient $\alpha$ keeps constant in one round of data transmission, but changes independently from one to another.

\section{Adaptive Network Code CoOperation}

Recently, network coding is getting increasing interest for a cooperative wireless network where a relay node plays the role of the network coding node which mixes the information received from other nodes and airs the coded ones, which can improve the overall system performance.

Exploiting the network coding technology and adapting to the changing network topology, ANCC can adequately combat the wireless fading, which significantly outperforms repetitionbased schemes and space-time coded cooperation frameworks.

The protocol works as follows. In the broadcast phase, each terminal broadcasts its wireless data packet (broadcast-packet) in its belonged time slot and all the others keep silence and decode what it hears. Since there exists random fading, the terminal may not be able to recover all the broadcast-packets. retrieval-set $\Re(i)$ denotes the assemble of correct-decoding broadcast-packets for terminal $i$, where $\Re(i) \subset\{1,2, \cdots, m\}$. In the relay phase, terminal $i$ randomly selects a small, fixed number (referred to $D$ ) of broadcast-packets from $\Re(i)$, and computes its check-sum (relay-packet) by XORing those packets symbol by symbol in the binary domain, then transmits the result to the destination in the designed time slot.

Therefore, through each communication round, a $(2 m, m)$ distributed network code in the form of a random, systematic, degree- $D$ low-density generate-matrix (LDGM) ${ }^{1}$ code, is generated at the destination. The systematic bits of the distributed LDGM code comprises the broadcast-packets transmitted in the broadcast phase, the parity bits are formed of the relaypackets sent in the relay phase.

Take a cooperative wireless network for an example, where 6 terminals, $S_{1}$ to $S_{6}$ and the inter-user channels form an instantaneous network topology as shown in Fig. 1 (destination is not shown in Fig. 1). If terminal $j$ decodes successfully the packet from source $i$, a directed edge, $i$ to $j$, is generated. The retrieval-set $\Re(i)$ of each terminal is, respectively,

$$
\begin{aligned}
& \Re(1)=\{1,2,4\}, \\
& \Re(2)=\{1,2, \mathbf{4}, \mathbf{5}, 6\}, \\
& \Re(3)=\{\mathbf{1}, 2,3, \mathbf{4}, 6\}, \\
& \Re(4)=\{1, \mathbf{3}, 4, \mathbf{5}, 6\}, \\
& \Re(5)=\{1, \mathbf{2}, 4,5, \mathbf{6}\}, \\
& \Re(6)=\{\mathbf{1}, \mathbf{2}, 3,5,6\} .
\end{aligned}
$$

\footnotetext{
${ }^{1} \mathrm{~A}$ terminal which is yet to relay may continue to decode and collect relaypackets in the relay phase and the correctly decoding relay-packets will be included in the retrieve-set. Then, low-triangular LDPC codes are generated at the destination. But it only can occur when the system operates in a TDMA manner. For simplicity, we only consider LDGM codes in this paper, which is suitable for any systems.
}

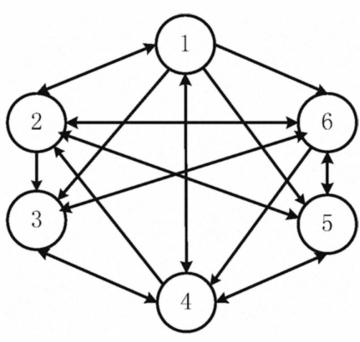

Fig. 1. an instantaneous network topology of 6 terminals communicating to a common destination (not shown)

The broadcast-packets marked in normal font represent those each terminal selects to form check-sum in the relay phase. So a parity check matrix of the network LDGM code is achieved:

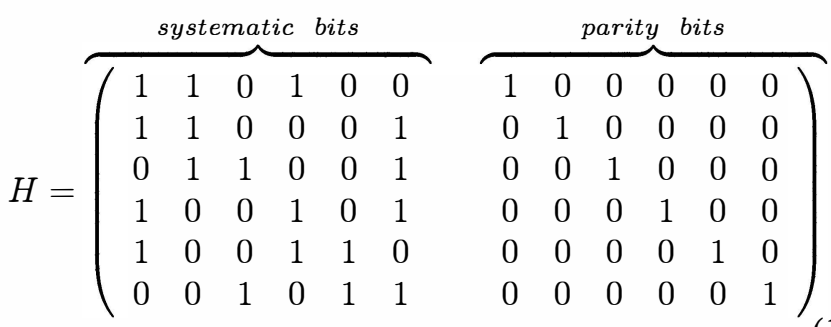

Because of the random construction of the code, the information that how the check-sums are formed (selection indication) needs to be included in the head of each relay-packet, thereby the destination can generate the adaptive decoder that matches the instantaneous code graph and perform messagepassing decoding algorithm, which can be implemented by software radio [14]. To sum up, the ANCC protocol is shown in Fig. 2(A). It is clear that different selections of the subset of $\Re(i)$ result in different distributed LDGM codes.

\section{SOURCE-DESTINATION NETWORK CODED COOPERATION(SDNCC)}

\section{A. The Basic Idea of SDNCC}

Cooperation among different users can make use of all dimensions of communication resources (i.e. time, frequency, spread code or terminal etc.). The ANCC protocol only takes advantage of the cooperation between terminals but not the overall system. Utilizing each source-destination CSI obtained by the destination for resource management, it is evident that it can bring in extra benefits. Furthermore, if the system complexity is not considered, terminals never stop to relay unless the destination successfully decodes all the broadcastpackets, that is to say, a rateless digital fountain code is generated [15]. However, two above advanced protocols are not economic.

The broadcast-packets in good channels need less or no error protection and those in bad channels should require greater protection, which can intuitively improve the system performance. Motivated by above idea, we propose Sourcedestination network coded cooperation(SDNCC), which makes use of the indexed feedback from the destination to indicate which terminal packets to form the check-sum and which 


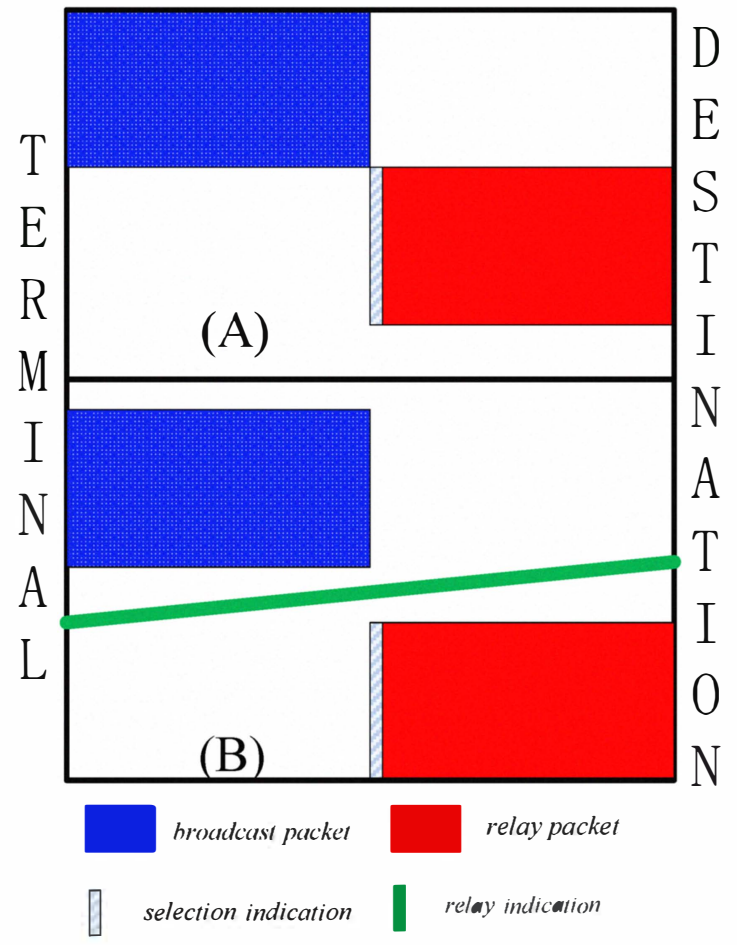

Fig. 2. (A)Adaptive network coded cooperation (ANCC) protocol. (B) Source-destination network coded cooperation(SDNCC) protocol.

terminals to send the check-sum in the relay phase. Thereby, LDGM codes with unequal error protection are generated at the destination.

Fig. 2(B) demonstrates the SDNCC strategy .The specific process works as below: In the broadcast phase, each terminal broadcasts its broadcast-packet while the others keep silent and decode what it hears. In the indication phase, according to the source-destination CSIs, the destination use relay indication, $\mathbf{R}$, to mark the first $l(1 \leq l \leq m)$ worst channels, thus broadcasts it to each terminal. $\mathbf{R}(i)(1 \leq i \leq l)$ represents the terminal whose broadcast-packet is allowed to be computed to form the check-sum. In the relay phase, each terminal randomly selects $D$ broadcast-packets from $\Re \cap \mathbf{R}$, Adds them by modulo 2 from symbol by symbol and airs the result to the destination in its own channel.

Thus a distributed $(m, 2 m)$ LDGM code is produced, whose parity check matrix has $m-l$ zero columns, that is to say, $m-l$ broadcast-packets achieve no error protection. Clearly, ANCC is a special case of SDNCC with $l=m$.

In practical projects, one terminal in $\mathbf{R}$ is marked by ' 1 ', otherwise ' 0 '. Thus, only one bit can represent which terminal packets will be selected for computing relay-packets. Compared with ANCC, SDNCC requires the destination to feedback $m$ bits for relay indication information, but the additional cost in the indication phase could be ignored if the length of broadcast-packet is large, which can be implemented easily and has great practical value.

\section{PER FORMANCE ANALYSIS}

Before analyzing simulation results, we study the outage probability of SDNCC. We first formulate the mutual in- formation of each terminal, then provide the corresponding outage probability as a function of the network size $m$ and the parameter $l$. Moreover, assuming $l$ is a linear function of $m$, at last we provide their numerical analysis with $m<\infty$ as well as their limit evaluation with $m \rightarrow \infty$.

\section{A. Preparations}

We begin with introducing some signs for convenient description. Here, $P(i \in \mathbf{R})$ denotes the channel quality of terminal $i$ is first $l$ worst in $m$ ones. We use subscript $(i, d)$ to denote the channel from terminal $i$ to the destination. Since all channels are considered independent identically and distributed, we can easily get

$$
P(i \in \mathbf{R})=\frac{l}{m}, \quad i=1,2, \ldots m
$$

Following the definition of $\mathbf{R}$, another expression can be written as:

$$
P(i \in \mathbf{R})=P\left(\left|h_{i, d}\right|^{2} \leq\left|h_{\mathbf{R}}\right|^{2}\right)
$$

where $h_{\mathbf{R}}$ is the maximal fading coefficient of the terminal in R.

From the statistical view ${ }^{2}$, each terminal belongs to $\mathbf{R}$ at the percentage of $\frac{l}{m}$. Thereby,

$$
P(i \in \mathbf{R})=P\left(\left|h_{i, d}\right|^{2} \leq\left|h_{\mathbf{R}}\right|^{2}\right)=\frac{l}{m}
$$

Using the pdf $p_{\left|h_{i, d}\right|^{2}}(x)=e^{-x}$, we compute

$$
\left|h_{\mathbf{R}}\right|^{2}=\ln \left(\frac{m}{m-l}\right)
$$

\section{B. Theoretical analysis of SDNCC}

We assume that perfect channel coding has been performed in each packet, thereby Shannon limitation can be used to denote the information that each terminal can convey per second. For simplicity, we focus on continuous-input, continuousoutput channels with Gaussian sources. After above preparations, we successively analyze the mutual information from terminal to the destination and then derive the outage probability of SDNCC.

1) Mutual Information: For terminal $i$ which gets no error protection in the relay phase, that is, $i \notin \mathbf{R}$. Using the Shannon formula with the instantaneous SNR, the mutual information from terminal $i$ to the destination can be directly written as ${ }^{3}$

$$
I_{i \notin \mathbf{R}}=\frac{1}{2} \log \left(1+\gamma\left|h_{i, d}\right|^{2}\right)
$$

where the factor $\frac{1}{2}$ accounts for: $2 m$ time slots are used in SDNCC, which constitutes of $m$ slots in the broadcast phase and $m$ slots in the relay phase. Thereby the contribution in broadcast phase is normalized by $\frac{1}{2}$ and that in relay phase is $\frac{1}{2}$.

In the relay phase of SDNCC, the broadcast-packets from the terminals $(i \in \mathbf{R})$ are encoded further into parity-check

\footnotetext{
${ }^{2} \mathrm{We}$ assume that $m$ is large enough for the application of law of large number and central limit theorem

${ }^{3}$ all the $\log ($.) functions in this paper have base 2
} 
packets of the LDGM code. Since the distributed codeword is transmitted by each terminal through independent channels in the relay phase, the total system mutual information can be written as the sum of the Shannon formula with all the instantaneous SNRs. We derive the following expression for the mutual information for terminal in $\mathbf{R}$ :

$$
\begin{aligned}
I_{i \in \mathbf{R}} & =\frac{1}{2} \log \left(1+\gamma\left|h_{i, d}\right|^{2}\right) \\
& +\frac{1}{2} \times \frac{m}{l} \times \frac{1}{m} \sum_{r=1}^{m} \log \left(1+\gamma\left|h_{r, d}\right|^{2}\right)
\end{aligned}
$$

where $\frac{m}{l} \times \frac{1}{m} \sum_{r=1}^{m} \log \left(1+\gamma\left|h_{r, d}\right|^{2}\right)$ can be explained as that the $m$ parity check packets of the network code protect $l$ terminals in $\mathbf{R}$ equally.

From (6) and (7), we can see that the mutual information in SDNCC is not a function of retrieval-set $\Re$, which is similar to ANCC. For reasonable $m$ and $l$, it is always true that the size of $\Re$ is larger than the fixed number $D$, which guarantees that the resulting distributed LDGM codes are excellent.

Following equation (4), (5), (6) and (7), for SDNCC, the mutual information of each terminal can be written as (8).

2) Outage probability: According to equation (8) and the definition of the outage probability, we can directly derive it, which can be seen in (9).

Following equation (9), we directly derive $\Gamma_{1}$ as

$$
P_{1}=\left\{\begin{array}{cc}
1-\exp \left[-\frac{1}{\gamma}\left(2^{2 R}-1\right)\right], & \gamma<\frac{\left(2^{2 R}-1\right)}{\ln (m / m-l)} \\
0 & \text { otherwise }
\end{array}\right.
$$

For $P_{2}$, we first define

$$
\begin{aligned}
& f_{1}=\frac{1}{2} \log (1+\gamma u), \\
& \left.f_{2}=\frac{1}{2 l} \log (1+\gamma u)\right]
\end{aligned}
$$

where the pdf of $u$ is $p_{u}(x)=e^{-x}, x \geq 0$. Using the Jacobi law, we can easily get the pdf of $f_{1}$ and $f_{2}$ :

$$
\begin{aligned}
p_{f_{1}}(y) & =p_{y}\left(f_{1}^{-1}(y)\right) \frac{\partial f_{1}^{-1}(y)}{\partial y_{2 y}} \\
& =\frac{2 \ln (2)}{\gamma} 2^{2 y} e^{\left(1-2^{(1)}\right) / \gamma} \\
p_{f_{2}}(y)= & p_{y^{\prime}}\left(f_{2}^{-1}(y)\right) \frac{\partial f_{2}^{-1}(y)}{\partial y} \\
& =\frac{2 l \ln (2)}{\gamma} 2^{2 l y} e^{\frac{1}{\gamma}\left(1-2^{2 l y}\right)}
\end{aligned}
$$

We then achieve

$$
P_{2}=\int_{0}^{R} p_{f_{1}}(y) \otimes \overbrace{p_{f_{2}}(y) \otimes p_{f_{2}}(y) \otimes \cdots \otimes p_{f_{2}}(y)}^{m} d y
$$

where $\otimes$ denotes the convolution operation. Thereby, the outage probability in SDNCC can be written as:

$$
P_{S D N C C}(R)=\frac{l}{m} P_{1}+\frac{m-l}{m} P_{2}
$$

(15) is almost impossible to simplify. Therefore we will numerically analyze it for different $l$, which is illustrated in Section V-C.

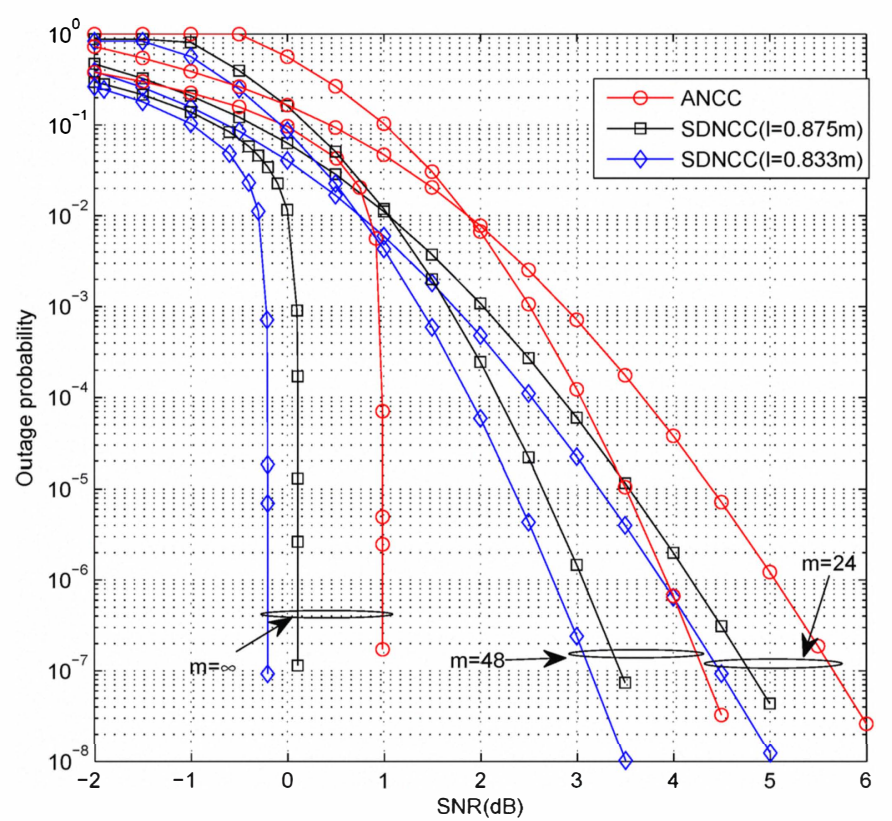

Fig. 3. Outage probability of SDNCC with different $l$ under different number of terminals and $s=m$

Next, we analyze the outage probability when the size of network $m$ gets close to infinity. From the law of large numbers, we get

$$
\begin{aligned}
\Omega & =\lim _{m \rightarrow \infty} \frac{1}{2} \times \frac{1}{l} \sum_{r=1}^{m} \log \left(1+\gamma\left|h_{r, d}\right|^{2}\right) \\
& =\frac{m}{2 l} E\left(\log \left(1+\gamma\left|h_{r, d}\right|^{2}\right)\right) \\
& =\frac{m}{2 l} \int_{0}^{\infty} \log (1+\gamma y) e^{-y} d y \\
& =m \exp \left(\frac{1}{\gamma}\right) E i\left(\frac{1}{\gamma}\right) /(2 l \ln (2))
\end{aligned}
$$

From (17), we can derive

$$
\begin{aligned}
\lim _{m \rightarrow \infty} P_{2} & =\operatorname{Pr}\left[\frac{1}{2} \log \left(1+\gamma\left|h_{s, d}\right|^{2}\right)+\Omega<R\right] \\
& =1-\exp \left[\frac{1}{\gamma}\left(1-2^{2 R-m \exp \left(\frac{1}{\gamma}\right) E i\left(\frac{1}{\gamma}\right) /(l \ln (2))}\right)\right],
\end{aligned}
$$

Therefore, we can get

$$
\lim _{m \rightarrow \infty} P_{S D N C C}(R)=\frac{l}{m} P_{1}+\frac{m-l}{m} \lim _{m \rightarrow \infty} P_{2}
$$

\section{Numerical results}

To demonstrate the superiority of SDNCC, in this subsection we numerically evaluate the outage probability of the two protocols. Since the the outage of SDNCC is a function of $m$ and $l$, we analyze the their contributions to its outage probability in Fig. 4. From Fig. 4, it is obvious that the outage of SDNCC and ANCC improves with the increase of $m$ and descends fast as the SNRs increase. As expected, the outage improves as $l$ decreases, which is attributed to the fact that the broadcast-packets in the worse channels receive more error protection as $l$ decreases. Whether $m$ is finite or not, numerical results show that SDNCC achieves more than $1 \mathrm{~dB}$ coding gain at the $10^{-7}$ outage probability. 


$$
I_{S D N C C}=\left\{\begin{array}{c}
\frac{1}{2} \log \left(1+\gamma\left|h_{i, d}\right|^{2}\right), \quad\left|h_{i, d}\right|^{2}>\ln \left(\frac{m}{m-l}\right) \\
\frac{1}{2} \log \left(1+\gamma\left|h_{i, d}\right|^{2}\right)+\frac{1}{2} \times \frac{1}{l} \sum_{r=1}^{m} \log \left(1+\gamma\left|h_{r, d}\right|^{2}\right), \quad 0<\left|h_{i, d}\right|^{2}<\ln \frac{m}{m-l},\left|h_{r, d}\right|^{2}>0
\end{array}\right.
$$

$$
P(R)=\left\{\begin{array}{c}
P_{1}=\operatorname{Pr}\left[\frac{1}{2} \log \left(1+\gamma\left|h_{i, d}\right|^{2}\right)<R\right], \quad\left|h_{i, d}\right|^{2}>\ln \left(\frac{m}{m-l}\right) \\
P_{2}=\operatorname{Pr}\left[\frac{1}{2} \log \left(1+\gamma\left|h_{i, d}\right|^{2}\right)+\frac{1}{2 l} \sum_{r=1}^{m} \log \left(1+\gamma\left|h_{r, d}\right|^{2}\right)<R\right], 0<\left|h_{i, d}\right|^{2}<\ln \frac{m}{(m-l)},\left|h_{r, d}\right|^{2}>0
\end{array}\right.
$$

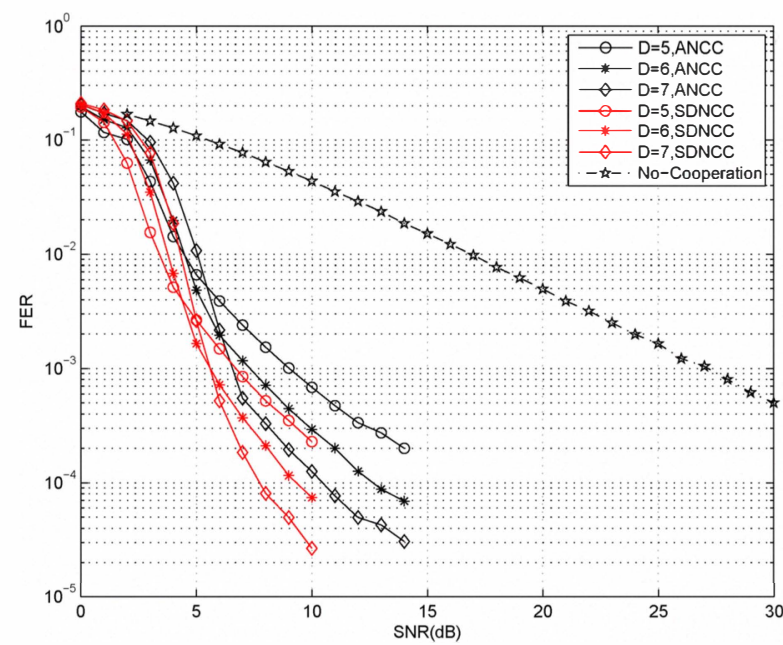

Fig. 4. Performance of SDNCC and ANCC. $m=300$

\section{Simulation results}

After theoretical analysis, we discuss the effectiveness of SDNCC through simulations. A large homogeneous wireless network with $m=300$ terminals communicating with a common destination is considered, where every channel has the same SNR. Belief Propagation algorithm with iteration 31 is adopted at the destination. Since each packet can use any practical channel code, different channel codes result in different bit error rate (BER) of the whole system. In order to evaluate the impact of user cooperation, we only focus on the performance after network coding and ignore the channel coding used in each packet. Thereby we assume each terminal transmits one bit for itself and relays one bit for others and the generated LDGM ensembles consists of tens of thousands of codes with codeword length 600 at the destination.

Compared with ANCC, the average performance of SDNCC with $l=250$ is illustrated in Fig. 4. The average SNR of channels between terminals and from terminals to destination is plotted in the $\mathbf{X}$ axis. For fairness, the total energy consumption of SDNCC and ANCC for cooperation round are all the half of that in the no-cooperation scheme. The $\mathbf{Y}$ axis denotes the FER averaged over different packets from different terminals. In Fig. 4, SDNCC with $l=250$ significantly outperforms ANCC at the same FER. The lower the FER, the more the encoding gain! For example, SDNCC with $D=7$ gets less than $2 \mathrm{~dB}$ gain over ANCC at the FER of
$10^{-3}$, while achieve more than $4 \mathrm{~dB}$ at the FER of $3 \times 10^{-5}$.

\section{CONCLUSION}

In this paper, a new user-cooperation scheme, termed source-destination network coded cooperation (SDNCC), is proposed for large wireless ad-hoc networks. Matching the quality of channels, the destination in SDNCC feedbacks which terminal packets to be selected for check-sum and distributed LDGM codes with unequal error protection codes are generated, which only costs 1 bit per terminal. For SNDCC, the closed-form expression of outage probability is derived for infinite networks and it is numerically analyzed when the network size is finite. Whether analysis or simulation results, SDNCC significantly outperforms ANCC.

\section{REFERENCES}

[1] E. C. van der Meulen, "Three-terminalcommunicationchannels," Adv. Appl.Prob.,vol. 3, pp. 120-154, 1971.

[2] R. Ahlswede, N. Cai, S.-Y.R. Li, and R. W. Yeung,"Network information flow," IEEE Trans. Inf. Theory, vol. 46, pp. 1204-1216, 2000.

[3] R. Koetter and M. M'edard, "An algebraic approach to network coding," IEEE/ACM Trans. Networking, vol. 11, no. 5, pp. 782-795, 2003

[4] $\mathrm{X}$. Bao and J. Li, "Matching code-on-graph with networks-on-graph: adaptive network coding for wireless relay networks," in Proc. Allerton Conf. on Commun., Control and Computing, Urbana Champaign, IL, Sept. 2005.

[5] X. Bao and J. Li, "Adaptive network coded cooperation(ANCC) for wireless relay network: matching code-on-graph with network-on-graph," IEEE Trans. wireless commun., vol.7, no.2, Feb.2008

[6] Andrea Montanari, "Tight bounds for LDPC and LDGM codes under MAP decoding," IEEE Trans. Inf. Theory, vol. 51, no. 9, Sept. 2005

[7] J. Laneman, "Cooperative Diversity in Wireless Network: Algorithm and Architecture", Ph.D. dissertation, Massachusetts Institute of Technology, Cambridge, MA, Aug. 2002

[8] J. Laneman and G. Wornell, "Distributed space-time-coded protocols for exploiting cooperative diversity in wireless networks," IEEE Trans. Inf. Theory, vol. 49, no. 10, pp. 2415-2425, Oct. 2003

[9] Y. Chen, S. Kishore, and J. Li, "Wireless diversity through network coding," in Proc. IEEE Wireless Commun. Networking Conf., March, 2006.

[10] G. Kramer, M. Gastpar, and P. Gupta, "Cooperative strategies and capacity theorems for relay networks," IEEE Trans. Inform. Theory,vol. 51, no. 9, Sep. 2006

[11] S. Cui, A. Haimovich, O. Somekh and H. Vincent Poor, "Opportunistic relaying in wirelss networks," IEEE Trans. Inf. Theory vol. 55, no. 11, pp. 5121-5137, Nov. 2009

[12] A. Bletsas, A. Khisti and M. Win, "Opportunistic cooperative diversity with feedback and cheap radios", IEEE Trans. Wireless Commun., vol. 7, no. 5, pp.1823-1827, may 2008

[13] C. Peng, Q. Zhang, M. Zhao, Y.Yao, "Opportunistic network-coded cooperation in wireless networks", in Proc. IEEE. Wireless Communications and Networking Conference (WCNC'07), pp. 3358-3363 Mar. 2007

[14] Joseph Mitola, "Software radio architecture: A mathematical perspective," IEEE J. Sel. Areas Commun., vol. 17, no. 4, April, 1999

[15] J. W. Byers, M. Lubu and M. Mitzenmacher, "A digital fountain approach to asynchronous reliable multicast," IEEE J. Sel. Areas Commun., vol. 20 , no. 8 , pp. $1528-1540$, Oct. 2002 